\title{
CNO Abundances in Magellanic Cloud OB Supergiants
}

\author{
Paul A. Crowther \\ Department of Physics \& Astronomy, UCL, Gower Street, London \\ WC1E $6 B T$, UK
}

Christopher J. Evans

The Isaac Newton Group of Telescopes, Apartado de Correos 321, 38700, Santa Cruz de La Palma, Canary Is., Spain

\begin{abstract}
We present CNO abundance determinations in 11 Magellanic Cloud $\mathrm{O}$ and early B supergiants based on physical parameters obtained from FUSE, HST/IUE and VLT spectroscopy, plus model atmospheres which allow for stellar winds and line blanketing. In all cases, nitrogen is substantially enhanced whilst carbon and generally oxygen are moderately depleted, indicative of rotationally induced mixing of unprocessed and CNO processed material at their stellar surfaces.
\end{abstract}

The recent availability of high quality UV, far-UV and optical spectroscopy of individual early-type stars in the Magellanic Clouds, together with sophisticated non-LTE modelling codes, now permit the determination of reliable physical parameters. Here, we present results obtained for a small sample of Large and Small Magellanic Cloud OB supergiants, emphasising their elemental abundances versus initial abundances as inferred from HII region studies (Russell \& Dopita 1992).

Individual targets are listed in Table 1, for which optical spectroscopy were obtained with the Very Large Telescope spectrograph UVES. Far-UV FUSE datasets were taken from the guaranteed hot star team program, whilst UV data were retrieved from the HST/IUE archive. Analysis is carried out using the non-LTE code of Hillier \& Miller (1998) which allows for spherical extension and line blanketing. Details of our approach followed are described in Crowther et al. (2002) and Hillier et al. (2003). All targets are apparently slow rotators with $60 \leq v \sin i \leq 120 \mathrm{~km} \mathrm{~s}^{-1}$.

We have established a major downward revision in the temperature scale for $\mathrm{O}$ supergiants relative to earlier studies which neglected stellar winds and blanketing. Our ultimate aim with this project is to empirically study the dependence of wind strength on spectral type and environment, although this requires study of a much larger sample. For the present topic, stellar parameters are fixed by optical $\mathrm{He} / \mathrm{Si}$ lines, permitting $\mathrm{CNO}$ abundances to be constrained, principally via NIII 4097 and CIII 4647-50, plus OII 4415-17 in late O and early B supergiants. It is clear from Table 1 that all OB stars are $\mathrm{N}$ rich, with modest $\mathrm{C}$ and $\mathrm{O}$ depletions. We attribute these abundance patterns to rotationally induced mixing of unprocessed and CNO processed material at their stellar surfaces. 
Table 1. $\quad \mathrm{CNO}$ abundances for Magellanic Cloud OB supergiants obtained by (a) Crowther et al.; (b) Hillier et al.; (c) Evans et al. relative to LMC/SMC HII regions according to Table 1 of Russell \& Dopita (1992).

\begin{tabular}{|c|c|c|c|c|c|c|}
\hline Star & Sp Type & galaxy & $\Delta \log (\mathrm{C} / \mathrm{H})$ & $\Delta \log (\mathrm{N} / \mathrm{H})$ & $\Delta \log (\mathrm{O} / \mathrm{H}$ & Ref \\
\hline HDE 269698 & O4 Iaft & LMC & -0.7 & +1.8 & $-0.75:$ & $\mathrm{a}$ \\
\hline HDE 270952 & O6 Iaf+ & LMC & -0.4 & +1.5 & -0.75 : & a \\
\hline $\mathrm{AzV} 232$ & O7 Iaf+ & SMC & -0.2 & +1.65 & -0.4 & $a$ \\
\hline $\mathrm{AzV} 83$ & O7 Iaf+ & SMC & -0.15 & +1.8 & -0.25 & $b$ \\
\hline $\mathrm{AzV} 372$ & O9 Iabw & SMC & -0.35 & +1.05 & .. & $c$ \\
\hline $\mathrm{AzV} 70$ & O9.5 Ibw & SMC & -0.6 & +1.25 & .. & $\mathrm{c}$ \\
\hline Sk-66 169 & O9.7 Ia+ & LMC & -0.2 & +1.15 & +0.0 & a \\
\hline HDE 269896 & ON9.7 $\mathrm{Ia}+$ & LMC & -0.7 & +1.6 & -0.6 & $\mathrm{c}$ \\
\hline HDE 269050 & B0 Ia & LMC & +0.0 & +1.4 & -0.1 & $\mathrm{c}$ \\
\hline $\mathrm{AzV} 235$ & B0 Iaw & SMC & -0.45 & +1.9 & +0.15 & $\mathrm{c}$ \\
\hline $\mathrm{AzV} 488$ & B0.5 Iaw & SMC & -0.6 & +1.45 & -0.2 & $\mathrm{c}$ \\
\hline
\end{tabular}

It is possible that absolute $\mathrm{CNO}$ abundances may be in error using our technique, but Hillier et al. (2003) derived apparently normal SMC abundances for $\mathrm{AzV} 69$ (OC 7.5III(f)) for which $\mathrm{C}, \mathrm{N}$ and $\mathrm{O}$ abundances were each within 0.2 dex of Russell \& Dopita (1992), adding weight to the present methods. Regardless of absolute uncertainties in abundance, HDE 269896 (ON 9.7Ia+) is of particular interest since its $\mathrm{N} / \mathrm{C}$ ratio is a factor of almost ten times larger than Sk-66 $169(\mathrm{O} 9.7 \mathrm{Ia}+)$. This adds quantiative support to previous claims that the progression from ' $\mathrm{O}$ ' to ' $\mathrm{ON}$ ' indicates a greater degree of $\mathrm{CNO}$ processing at the surface. Similar analyses of Galactic OB supergiants are presently underway for differential abundance studies. Venn (1999) has previously found SMC A supergiants to be strongly $\mathrm{N}$-rich (up to $+1.2 \mathrm{dex}$ ) relative to HII regions, whilst Lennon et al. (1991) obtained an identical CNO pattern to that presented here for $\mathrm{AzV} 488$ using an alternative technique.

Acknowledgments. Many thanks to John Hillier for making available his model atmosphere code, to Alex Fullerton for careful FUSE reductions, and to the Royal Society and PPARC for financial support.

\section{References}

Crowther P.A., Hillier D.J., Evans C.J. et al. 2002, ApJ 579, 774

Evans C.J., Crowther P.A., Fullerton A.W., Hillier D.J., 2003, MNRAS 588, 1039

Hillier D.J., Miller D.L., 1998, ApJ, 496, 407

Hillier D.J., Lanz T. Heap S.R. et al. 2003, ApJ in press

Lennon D.J., Becker S. T., Butler K. et al. 1991, A\& 252, 498

Russell S.C., Dopita M.A, 1992, ApJ 384, 508

Venn K., 1999, ApJ 518, 405 